\title{
Gross dissection of the human brain
}

\author{
LENNART HeIMER, M.D. \\ Departments of Neurological Surgery and Neuroscience, University of Virginia Health Science \\ Center, Charlottesville, Virginia
}

Galen, who lived in the second century A.D., disputed the cardiocentric theory of Aristotle and instead proposed that the brain rather than the heart was the central organ of the body responsible for perceptual and intellectual functions. Galen was a strong proponent of brain dissections, but he used only animal brains because of widespread prejudice against dissections of the human body. This lack of scientific study of the human body persisted throughout the long, dark period of the Middle Ages.

In the late 15 th and early 16 th centuries, several anatomists and painters, including Leonardo da Vinci, the ultimate Renaissance man, defied the longstanding code against dissections of the human body because of their desire to learn more about its internal structure. It remained for Andreas Vesalius, however, to take the giant step toward a systematic study of human anatomy by means of dissection. The significance of Vesalius' masterpiece, $D e$ Humani Corporis Fabrica, published in 1543, was profound. Emerging from the superstitions and prejudices of the Dark Ages, Vesalius' book marks the beginning of a new epoch; observation of the structure of the human body during macroscopic dissection became the cornerstone of anatomical research and teaching. Rembrandt, in one of his most famous paintings, the Anatomy Lesson of Professor Tulp, immortalized the excitement and fascination generated by the dissection of a cadaver almost 400 years ago.

Macroscopic dissections of the human brain remained a major scientific method well into the nineteenth century. In the beginning, the brains were sliced in different directions, but because the functions of this organ are in large part dependent on the manner in which its different parts are connected, it was difficult to obtain a general notion of how the various functions were accomplished, regardless of how the brain was sliced. The brilliant idea to follow the white matter fiber bundles in the direction of the fibers, apparently conceived by the Danish anatomist Steno in the second half of the 17th century, had a lasting impact on brain science. The so-called "teasing method" or blunt dissection of major fiber bundles marks the beginning of the field of tract-tracing, which more than any other research methodology has advanced our understanding of the functional-anatomical organization of the central nervous system. Incidentally, tract-tracing in the broadest sense of the term is still the mainstay of neuroscience strategies. This includes studying all aspects of neuronal circuitry, often in experimental animals, by using light and electron microscopic anatomical methods, as well as physiological, histochemical, and molecular biological techniques.

The technique of scraping away fibers in the direction of the fiber bundles was greatly facilitated when hardening of the brains in a fixation solution became more widely used in the nineteenth century. The stage was now set for several generations of pioneering European neuroanatomists to map the major fiber tracts in the brain and spinal cord. Hardening of the jellylike brain substance, usually with formalin, continues to be a universally accepted pretreatment for most macro- and microscopic studies of the brain and spinal cord.

The importance of macroscopic brain dissection as a research tool gradually declined as experimental techniques, histological staining methods, and serial sectioning of brain tissue were developed in the second half of the nineteenth century. Even during the twentieth century, however, important discoveries were made with the aid of blunt dissections of human brains. Curran's description of the occipitofrontal fasciculus in the Journal of Comparative Neurology and Psychology in 1909 comes to mind, and important observations regarding the optic radiation and the Meyer loop were published in the Anatomical Record by Rasmussen as late as 1943 .

An even more recent demonstration of the value of the blunt dissection of fiber tracts is provided by Türe and his colleagues, who published a paper in Neurosurgery in 1997 that challenged the existence of a superior occipitofrontal fasciculus, believed to be located at the lateral 
margin of the lateral ventricle underneath the corpus callosum. Needless to say, gross anatomical dissection of myelinated fiber bundles cannot disprove the existence of a superior occipitofrontal fasciculus, but Türe's elegant study was a timely reminder of how ill equipped we still are to trace connections from their origin to their terminations in the human brain, especially if the connections are poorly myelinated or unmyelinated. To facilitate the dissection of myelinated fiber bundles, Türe and colleagues used the Klingler freeze-thaw method (freezing of formalin-fixed brains for 1 week at -10 to $-15^{\circ} \mathrm{C}$, followed by thawing in water). In the hands of experts with patience, the Klingler freeze-thaw method can provide exquisite pictures of major myelinated fiber bundles (see the article by Yaşargil, et al., in this issue of Neurosurgical Focus).

In vivo tractography performed using the new method of diffusion tensor (DT) imaging has become the new buzzword in clinical neuroscience. This method is based on the tendency of water molecules to diffuse in the direction of myelinated fiber bundles, and the results obtained in the living human brain are spectacular. Although in vivo tractography is already having an impact in many fields of clinical neuroscience, it is unrealistic to expect that new pathways defined by origin and termination will be discovered in the human brain by using the technique of DT imaging.
Only white matter bundles can be visualized and intracortical connections cannot be reconstructed. Whatever limitations DT imaging may have in discovering new pathways, the technique has provided yet another incentive to promote blunt dissection of myelinated fiber tracts in the human brain.

The aim of the "Gross Dissection" part of this issue of Neurosurgical Focus is to reinforce the value of classic macroscopic dissection as a vehicle for teaching functionally and clinically relevant brain anatomy. No book, plastic model, or high-tech computer simulation can replace gross dissections as a vehicle for the study of the human brain, and neurosurgeons and neuroradiologists, in particular, are actively promoting blunt dissection of white matter fiber bundles as part of their education and routine activities. No method can provide a better and more easily obtainable overview of the anatomical organization of the human brain than that of blunt dissection. A Yaşargil quote from his article in the November 2004 issue of the Journal of Neurosurgery may serve as an added incentive for promoting the technique of fiber dissection in neurosurgical education: "We [neurosurgeons] are charged with remembering that the white matter of the brain may not be likened to an ocean, where one may navigate freely." 